\title{
Nasopharyngeal chordoma in a patient with a severe form of sleep-disordered breathing: A case report
}

\author{
JOANNA RADZIKOWSKA ${ }^{1}$, ZUZANNA GRONKIEWICZ ${ }^{1}$, ANDRZEJ KUKWA ${ }^{2}$, WOJCIECH LISIK ${ }^{3}$, \\ ANNA M. CZARNECKA ${ }^{4}$, ANTONI KRZESKI ${ }^{1}$ and WOJCIECH KUKWA ${ }^{1}$ \\ ${ }^{1}$ Department of Otorhinolaryngology, Medical University of Warsaw, Warsaw 00-739; \\ ${ }^{2}$ Department of Otolaryngology and Head and Neck Disease, University of Varmia and Mazuria School of Medicine, \\ Olsztyn 10-082; ${ }^{3}$ Department of General and Transplantation Surgery, Medical University of Warsaw, \\ Warsaw 02-005; ${ }^{4}$ Department of Oncology, Military Institute of Medicine, Warsaw 04-141, Poland
}

Received May 19, 2014; Accepted January 22, 2015

DOI: 10.3892/ol.2015.3393

\begin{abstract}
Nasopharyngeal chordoma is a rare type of malignant neoplasm that originates in the remnants of the notochord, a primitive tissue of embryonic origin preserved outside the axial skeleton. Approximately one-third of chordomas are located in the base of the skull, in the midline of the body. The slow growth rate of the tumor, which gradually fills the nasopharyngeal cavity, contributes to a delayed oncological diagnosis. Among its isolated and non-specific symptoms, the obstruction of the nasopharynx is dominant, thus, sleep-disordered breathing (SDB) may occur. The current study presents the case of a 32-year-old female patient who was incidentally diagnosed with a nasopharyngeal chordoma during a diagnostic examination for SDB. The diagnostic examination was performed as a part of a research program for pathologically obese patients who qualified for bariatric surgery. Following tumor resection, a significant improvement in various polysomnographic parameters occurred, including a decrease in the apnea hypopnea index from 53.5 to 6.4 and an increase in the mean saturation rate from 92.5 to $95 \%$, confirming that an association exists between tumor obstruction of the nasopharynx and SDB. The incidental diagnosis of this rare type of neoplasm drew attention to diagnostic and therapeutic problems associated with nasopharyngeal chordomas. Furthermore, it indicated the necessity for the accurate laryngological examination of patients with SDB.
\end{abstract}

\section{Introduction}

Sleep-disordered breathing (SDB) describes a group of disorders that are characterized by breathing difficulties while

Correspondence to: Dr Wojciech Kukwa, Department of Otorhinolaryngology, Medical University of Warsaw, 19/25 Stepinska Street, Warsaw 00-739, Poland

E-mail: wojciechkukwa@gmail.com

Key words: chordoma, nasopharyngeal tumor, sleep-disordered breathing sleeping. Examples of SDB include primary snoring, upper airway resistance syndrome and obstructive sleep apnea (OSA). SDB is characterized by repeated narrowing of the upper airway during sleep, which leads to partial or complete obstruction of the airways $(1,2)$. The symptoms of SDB are dependent on the time of day; symptoms experienced at night include choking, pauses in breathing and nocturia, whereas day time symptoms include fatigue, extreme tiredness and a depressed mood (3). A number of predisposing factors have been identified, such as age ( $>40$ years), obesity, recent weight gain, male gender, alcohol consumption, nasal blockage and maxillo-mandibular abnormalities (1).

Nasopharyngeal organic lesions, particularly malignant neoplasms, are a rare cause of SDB in adults. Chordomas are histologically benign but clinically malignant axial skeleton neoplasms that represent $0.1-0.2 \%$ of all intracranial neoplasms, $6-16 \%$ of skull base neoplasms and 1-4\% of primary malignant bone tumors $(4,5)$. Furthermore, approximately one-third of all chordomas are located within the skull base $(6,7)$. The slow growth of chordomas and a lack of specific initial symptoms may prevent the diagnosis of the neoplasm, thus resulting in an extended period of tumor development. Clinically, with the exception of nasal patency disorders, chordoma symptoms may include headaches, diplopia, swallowing disorders, Eustachian tube patency disorders, tinnitus and secretion retention in the nasal cavity. The subtle onset and benign macroscopic image of the tumor (similar to an enlarged adenoid) may result in diagnostic errors.

The current study presents a case of incidentally diagnosed nasopharyngeal chordoma that manifested as a patency disorder in the upper airways and was the predominant cause of a severe form of SDB. Written informed consent was obtained from the patient.

\section{Case report}

The current study presents the case of a 32-year-old female patient, with a severe form of SDB caused by the presence of a nasopharyngeal tumor impairing nasal breathing. The patient was diagnosed with SDB at the Department of 
A

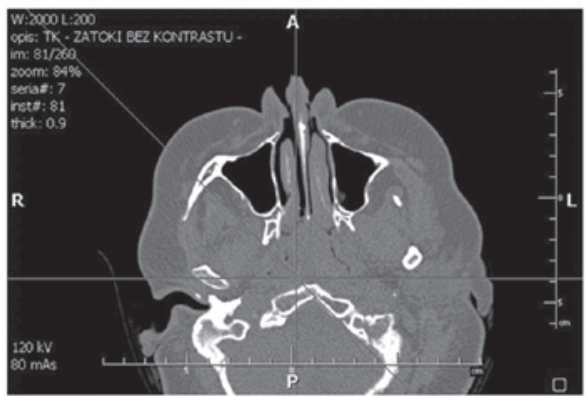

C

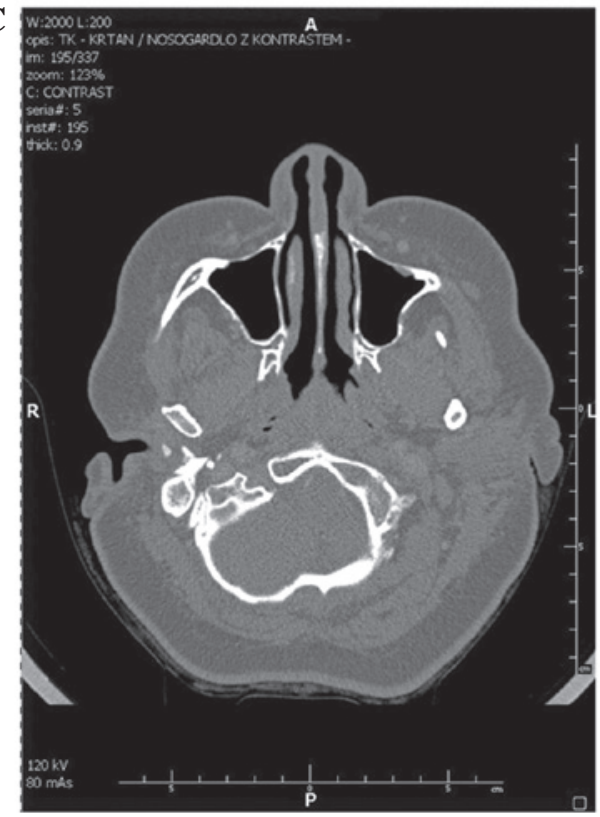

B

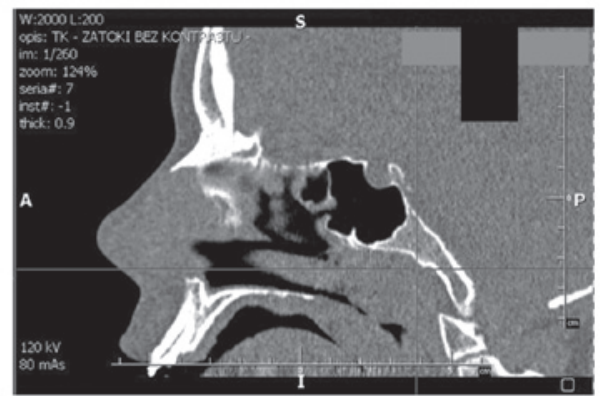

D

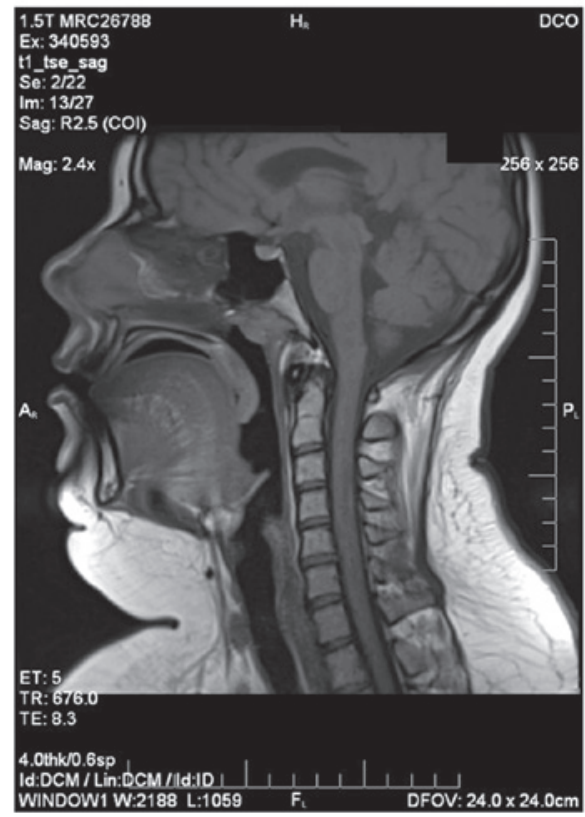

Figure 1. (A and B) Pre-operative computed tomography (CT) scan of the maxillofacial region. Soft tissue filling the nasopharynx was observed, but no evidence of sphenoid bone destruction was present. (C) Post-operative CT of the maxillofacial region with contrast demonstrating thickening of the posterior pharyngeal wall, but with no evidence of bone infiltration. (D) Post-operative magnetic resonance imaging examination indicating a contrast-enhanced pathological mass at the nasopharyngeal roof.

Otorhinolaryngology at the English Dentistry Division of the Medical University of Warsaw (Warsaw, Poland) during the course of a research program investigating a group of pathologically obese patients who qualified for bariatric surgery.

In accordance with the research program's assumptions, each patient routinely completed an interview, and underwent a laryngological physical examination, upper airway endoscopy, anterior rhinomanometry, computed tomography (CT) scan of the maxillofacial region and a full in-laboratory polysomnography. The present patient was diagnosed with third-degree obesity, with a body mass index (BMI) of $41.9 \mathrm{~kg} / \mathrm{m}^{2}$ (height, 1.62 meters; body weight, $110 \mathrm{~kg}$ ). During the interview, a five-year history of nasal patency disorders was noted. The laryngological examination revealed a left-sided deviation of the nasal septum to a limited degree and moderate hypertrophy of the tonsils. The isthmus of the pharynx was not significantly narrowed, however, an upper airway endoscopy revealed a soft-tissue mass with a smooth surface located within the nasopharynx, which caused almost complete impaired patency. Significant nasopharyngeal obstruction prevented an objective assessment of nasal ventilation with the use of anterior rhinomanometry. CT of maxillofacial region was consistent with the nasopharynx being filled with soft tissue and did not reveal features of destruction within the sphenoid bone (Fig. 1A and B).

A complete polysomnography, including electroencephalography, electrooculography and electromyography, was performed to assess the sleep structure of the patient. An assessment of respiratory function was performed by recording chest and abdomen respiratory movements, nasal and mouth air passage and continuous arterial blood oxygen saturation levels. In addition, continuous electrocardiogram records were kept. In the course of the polysomnography study, the patient exhibited 392 incidents of SDB (five obstructive, 19 central and 16 mixed apneas, and 352 hypopneas) resulting in an apnea hypopnea index (AHI) of 53.5. Furthermore, 367 desaturation events were recorded, with an average saturation of $92.5 \%$ and a minimum saturation of $72.5 \%$. Thus, a severe form of SDB was diagnosed. The patient qualified for surgical treatment with the aim of restoring nasal patency and histopathologically verifying the presence of a soft-tissue mass located within the nasopharynx. The lesion was removed using Beckman's adenotome and histopathologically assessed to determine a diagnosis of nasopharyngeal chordoma. Microscopic examination revealed a lobulated tumor divided by fibrous septa, composed of vacuolated neoplastic cells in a myxoid stroma. 
The resection was not microscopically radical, therefore, additional diagnostic and oncological treatments were planned. However, the patient did not agree to the proposed treatment strategies. Six months after the primary surgical procedure, the patient underwent a follow-up imaging assessment. CT of the maxillofacial region with contrast revealed thickening of the posterior pharyngeal wall on the border of the nasopharynx and oropharynx, with greater enlargement on the left side (Fig. 1C). The thickness of the pre-spinal tissue at the C2 level had increased to $\sim 13 \mathrm{~mm}$. The described area was contrast-enhanced within 63-80 HU limits and it was identified that the lesion adhered to the longissimus capitis muscle, however, unambiguous features of its infiltration were not identified in the CT images. In addition, the neck lymph nodes were not enlarged and no pathological remodeling within the bony structures was identified. Magnetic resonance imaging (MRI) examination clarified the presence of a nodular structure outlined within the nasopharyngeal roof, situated further towards the left side and unevenly contrast enhanced (Fig. 1D). Again, surgical and oncological treatment strategies were proposed to the patient, however, the patient refused. Six months after the chordoma diagnosis, polysomnography was performed as a follow-up examination and a significant improvement was identified in the respiratory parameters. Only 35 respiratory incidents of desaturation were recorded, and no apneas were detected. Furthermore, the AHI was 6.4, the average saturation during examination was $95 \%$ and the minimum saturation was $81 \%$.

\section{Discussion}

It is estimated that between 9 and $24 \%$ of the general population have SDB (8). Various risk factors for developing SDB exist, including obesity, the male gender, older age, a positive family history, hormonal disorders, tobacco smoke, alcohol consumption, maxillofacial region structural abnormalities and upper airway patency disorders. A recognized association exists between SDB and obesity, with SDB diagnosed in 50-77\% of obese patients (9). The probability of SDB occurrence rises with the increase in the degree of obesity; in particular, the risk of sleep apnea syndrome increases by 10 -fold among individuals with at least first-degree obesity (BMI, $>30 \mathrm{~kg} / \mathrm{m}^{2}$ ) (10). Furthermore, an obstructive form of SDB qualifying for surgical obesity treatment is diagnosed in 70-91\% of pathologically obese patients (BMI, $>40 \mathrm{~kg} / \mathrm{m}^{2}$ ) (11-13). The type of fatty tissue distribution is of particular importance in the SDB pathogenesis of obese patients (14-16). Deposits of fatty tissue within the neck compress the walls of the pharynx from the outside, and fatty tissue infiltrates degenerate the neck muscles, impairing the ability of the muscles to support the upper airways; thus, patency is more easily impaired in obese patients, particularly in those with a high fat distribution in the neck (17).

Upper airway patency disorders resulting from structural abnormalities, which are the result of various pathologies within the head and neck, are important in the pathogenesis of SDB (18). Thorough investigation into upper airway patency at the oropharynx has identified a number of factors involved in oropharynx obstruction, including a lengthened soft palate, tongue and uvula hypertrophy, and palatine tonsil hypertrophy (19). However, the role of nose and nasopharynx patency in SDB pathogenesis (with the exception of in children, where adenoids are the predominant cause of SDB) has yet to be discussed (20).

The lower section of the upper airways, in contrast to the nasal cavity, larynx, trachea and bronchi level, does not have cartilaginous or osseous scaffolding to provide rigid support (21). Instead, stabilization at this level is predominantly provided by the pharynx, palate and tongue muscles. During inspiration, a partial or complete collapse and narrowing of the lumen of the airway occurs when the negative pressure in the airway is greater than the tension of the stabilizing muscles of the wall. As a consequence, OSA occurs (19-22). The pathologies that lead to nasal patency impairment cause a rise in negative pressure in the upper airways during inspiration (23), therefore, increasing the risk of obstructive apneas. Nasal patency disorders may result from numerous anatomical abnormalities and pathologies that make nasal ventilation difficult, such as deviation of the nasal septum, nasal polyps, inferior nasal turbinate hypertrophy, middle nasal turbinate pneumatization, posterior nares underdevelopment, neoplasms, granulomatous diseases, adenoid hypertrophy, meningocele, foreign bodies, post-operative and post-traumatic adhesions, and allergic and non-allergic rhinitis (20,22).

Various studies in the literature have indicated that nasal patency disorders should be treated as a potential risk factor for SDB development $(24,25)$. For example, in a prospective study by Lofaso et al (26), it was stated that nasal patency disorders assessed on the basis of posterior rhinomanometry are independent risk factors for OSA development, although their impact was significantly weaker compared with the effect of obesity or facial bone abnormalities. By contrast, Young et al (27) did not identify a strong association between increased airway resistance at the level of the nasal cavity and SDB in the total population. In addition, the use of surgical treatment for nasal patency disorders as a treatment strategy for SDB is controversial, with contrary perspectives reported in the literature. Sériès et al (28) determined that surgical correction of nasal patency disorders is an effective method of SDB treatment, but only in patients who were not diagnosed with significant abnormalities of the facial bones. Isolated studies have described cases of upper airway neoplastic lesions, which predominantly manifest as nasal and nasopharyngeal obstruction, leading to SDB (29-31). Furthermore, Piccin and Sorrenti (29) described a retropharyngeal space lipoma with severe symptoms typical of SDB that completely subsided following surgical removal of the tumor.

The case described in the current study concerns a rare nasopharyngeal chordoma that manifested as a nasal obstruction, resulting in SDB. Chordomas are slow-growing malignant neoplasms originating from the remnants of the notochord, a primitive tissue of embryonic origin that is preserved outside of the axial skeleton. Chordomas develop in the midline of the body, most often in the sacrococcygeal area of the spine (50-60\%), the skull base $(25-30 \%)$, the cervical spine $(\sim 10 \%)$ and the thoracolumbar segment of the spine $(\sim 5 \%)$. Chordoma localization in the skull base is limited to the clivus, and casuistic cases are concerned with lesions developing outside of the bones as a soft-tissue mass in the nasopharynx $(32,33)$. In addition, chordomas constitute only $0.2 \%$ of all nasopharyngeal 
neoplasms, therefore, diagnosis poses a significant challenge, and pre-surgical diagnostics based on a patient's clinical condition and imaging examinations do not typically coincide with the final histopathological diagnosis. Chordoma can develop at any age, however, it is predominantly diagnosed in adults. Lesions in the skull base typically occur in the third and fourth decade of life, while tumors of the sacrococcygeal area typically occur in the fifth and sixth decade (34). Furthermore, male individuals suffer from chordoma twice as often as females (35). Chordomas leading to distant metastasis to the lungs, liver, bones and lymph nodes have previously been described in the literature (36).

Nasal patency symptoms are dominant in tumors located within the nasopharynx. Imaging diagnostics are based on CT and MRI scans of the area occupied by the tumor, however, the two techniques provide non-specific images of the lesion (37). Chordomas are not chemotherapy-sensitive tumors, therefore, the typical treatment strategy is radical surgery. However, the localization of chordomas in the vicinity of nervous system structures makes negative tissues margins difficult or even impossible to obtain. In such cases, surgical resection should be complemented with post-operative radiotherapy. Efficient chordoma radiotherapy treatment requires the use of high-dose radiation ( $>60 \mathrm{~Gy}$ ), which may damage sensitive tissue in the brain and optic nerve chiasm area. Promising alternatives include modern conformal radiotherapy techniques, intensity-modulated radiation therapy and proton beam therapy (38). Furthermore, combined treatment strategies provide good local control. It is estimated that the five-year survival rate of patients with chordomas of all localizations is $\leq 70 \%$ (39).

The nasopharyngeal chordoma described in the current study was incidentally diagnosed due to poor symptomatology. The chordoma was diagnosed during SDB diagnostics performed as a part of a research project of pathologically obese patients qualifying for bariatric surgery. A non-typical radiogram without features of adhering bone structure destruction and endoscopic images imitating nasopharyngeal lymphatic tissue hypertrophy were obtained, thus, the SDB diagnostics were not extended to incorporate neoplastic disease at the preliminary stage. A polysomnographic control examination, which was performed independently of oncological proceedings, revealed a significant improvement in various respiratory parameters following tumor removal; however, the BMI did not change, as the patient was not subjected to bariatric surgery. Thus, it is proposed that the obstruction of the nasopharynx by the tumor was the predominant cause of the SDB.

SDB is a common phenomenon in pathologically obese individuals and polysomnography should be the diagnostic gold standard in this group of patients. Although the predominant risk factor in SDB patients with a high BMI is the obesity itself, this does not prejudice the duty of additional laryngological diagnostics with the aim of excluding organic and anatomical causes of SDB.

Laryngological assessment of patients with SDB should be based on a detailed interview and physical examination, followed by an endoscopic examination of the upper airways. When necessary, this should be accompanied by a head and neck imaging examination.
In conclusion, nasopharyngeal chordoma is a rare type of slow-growing nasopharyngeal malignant neoplasm that may remain unnoticed for a long period of time. Due to its non-specific clinical presentation and, in a number of cases, a lack of radiological features consistent with infiltration of the nasopharyngeal bone structures, this neoplasm is often misdiagnosed as a benign lesion of the nasopharynx, such as an enlarged adenoids.

\section{Acknowledgements}

This study was supported by the EEA/Norway Grants (grant number POL/NOR/196258/2013.

\section{References}

1. Taranto Montemurro L and Kasai T: The upper airway in sleep-disordered breathing: UA in SDB. Minerva Med 105: 25-40, 2014.

2. Budhiraja R, Budhiraja P and Quan SF: Sleep-disordered breathing and cardiovascular disorders. Respir Care 55: 1322-1332; discussion, 1330-1332, 2010.

3. Ioachimescu OC and Collop NA: Sleep-disordered breathing. Neurol Clin 30: 1095-1136, 2012.

4. McMaster ML, Goldstein AM, Bromley CM, et al: Chordoma: incidence and survival patterns in the United States, 1973-1995. Cancer Causes Control 12: 1-11, 2001.

5. Kłosiński P, Lisiecki J, Goździewicz J and Kowalska B: Chordoma - treatment and prognosis. Współczesna Onkologia 7: 107-114, 2003 (In Polish).

6. Harbour JW, Lawton MT, Criscuolo GR, Holliday MJ, Mattox DE and Long DM: Clivus chordoma: a report of 12 recent cases and review of the literature. Skull Base Surg 1: 200-206, 1991.

7. Heffelfinger MJ, Dahlin DC, MacCarty CS and Beabout JW: Chordomas and cartilaginous tumors at the skull base. Cancer 32: 410-420, 1973.

8. Young T, Palta M, Dempsey J, Skatrud J, Weber S and Badr S: The occurrence of sleep-disordered breathing among middle-aged adults. N Engl J Med 328: 1230-1235, 1993.

9. Vgontzas AN, Tan TL, Bixler EO, Martin LF, Shubert D and Kales A: Sleep apnea and sleep disruption in obese patients. Arch Intern Med 154: 1705-1711, 1994.

10. Kyzer S and Charuzi I: Obstructive sleep apnea in the obese. World J Surg 22: 998-1001, 1998.

11. Frey WC and Pilcher J: Obstructive sleep-related breathing disorders in patients evaluated for bariatric surgery. Obes Surg 13: 676-683, 2003.

12. O'Keeffe T and Patterson EJ: Evidence supporting routine polysomnography before bariatric surgery. Obes Surg 14: 23-26, 2004.

13. Hallowell PT, Stellato TA, Schuster M, et al: Potentially life-threatening sleep apnea is unrecognized without aggressive evaluation. Am J Surg 193: 364-367, 2007.

14. Schäfer H, Pauleit D, Sudhop T, et al: Body fat distribution, serum leptin, and cardiovascular risk factors in men with obstructive sleep apnea. Chest 122: 829-839, 2002.

15. Shelton KE, Woodson H, Gay S and Suratt PM: Pharyngeal fat in obstructive sleep apnea. Am Rev Respir Dis 148: 462-466, 1993.

16. Horner RL, Mohiaddin RH, Lowell DG, et al: Sites and sizes of fat deposits around the pharynx in obese patients with obstructive sleep apnoea and weight matched controls. Eur Respir J 2: 613-622, 1989.

17. Schwartz AR, Patil SP, Laffan AM, et al: Obesity and obstructive sleep apnea: pathogenic mechanisms and therapeutic approaches. Proc Am Thorac Soc 5: 185-192, 2008.

18. Fogel RB, Malhotra A and White DP: Sleep. 2: Pathophysiology of obstructive sleep apnoea/hypopnoea syndrome. Thorax 59: 159-163, 2004.

19. Ryan CM and Bradley TD: Pathogenesis of obstructive sleep apnea. J Appl Physiol (1985) 99: 2440-2450, 2005.

20. Rappai M, Collop N, Kemp S and deShazo R: The nose and sleep-disordered breathing: what we know and what we do not know. Chest 124: 2309-2323, 2003. 
21. Rombaux P, Liistro G, Hamoir M, et al: Nasal obstruction and its impact on sleep-related breathing disorders. Rhinology 43 : 242-250, 2005.

22. Kohler M, Bloch KE and Stradling JR: The role of the nose in the pathogenesis of obstructive sleep apnoea and snoring. Eur Respir J 30: 1208-1215, 2007.

23. Anch AM, Remmers JE and Bunce H III: Supraglottic airway resistance in normal subjects and patients with occlusive sleep apnea. J Appl Physiol Respir Enveron Exerc Physiol 53: 1158-1163, 1982.

24. Suratt PM, Turner BL and Wilhoit SC: Effect of intranasal obstruction on breathing during sleep. Chest 90: 324-329, 1986.

25. Olsen KD, Kern EB and Westbrook PR: Sleep and breathing disturbance secondary to nasal obstruction. Otolaryngol Head Neck Surg 89: 804-810, 1981.

26. Lofaso F, Coste A, d'Ortho MP, et al: Nasal obstruction as a risk factor for sleep apnoea syndrome. Eur Respir J 16: 639-643, 2000.

27. Young T, Finn L and Kim H: Nasal obstruction as a risk factor for sleep-disordered breathing. The University of Wisconsin Sleep and Respiratory Research Group. J Allergy Clin Immunol 99: S757-S762, 1997.

28. Sériès F, St Pierre $S$ and Carrier G: Surgical correction of nasal obstruction in the treatment of mild sleep apnoea: importance of cephalometry in predicting outcome. Thorax 48: 360-363, 1993.

29. Piccin O and Sorrenti G: Adult obstructive sleep apnea related to nasopharyngeal obstruction: a case of retropharyngeal lipoma and pathogenetic considerations. Sleep Breath 11: 305-307, 2007.

30. Di Girolamo S, Marinelli L, Galli A and Ottaviani F: Retropharyngeal lipoma causing sleep apnea syndrome. J Oral Maxillofac Surg 56: 1003-1004, 1998.
31. Hockstein NG, Anderson TA, Moonis G, et al: Retropharyngeal lipoma causing obstructive sleep apnea: case report including five-year follow-up. Laryngoscope 112: 1603-1605, 2002.

32. Nguyen RP, Salzman KL, Stambuk HE, Ahuja AT and Harnsberger HR: Extraosseous chordoma of the nasopharynx. AJNR Am J Neuroradiol 30: 803-807, 2009.

33. DiFrancesco LM, Davanzo Castillo CA and Temple WJ: Extra-axial chordoma. Arch Pathol Lab Med 130: 1871-1874, 2006.

34. Klingler L, Trammell R, Allan DG, Butler MG and Schwartz HS: Clonality studies in sacral chordoma. Cancer Genet Cytogenet 171: 68-71, 2006.

35. Mizerny BR and Kost KM: Chordoma of the cranial base: the McGill experience. J Otolaryngol 24: 14-19, 1995.

36. Weber AL, Brown EW, Hug EB and Liebsch NJ: Cartilaginous tumors and chordomas of the cranial base. Otolaryngol Clin North Am 28: 453-471, 1995.

37. Yan ZY, Yang BT, Wang ZC, Xian JF and Li M: Primary chordoma in the nasal cavity and nasopharynx: CT and MR imaging findings. AJNR Am J Neuroradiol 31: 246-250, 2010.

38. Hug EB, Loredo LN, Slater JD, et al: Proton radiation therapy for chordomas and chondrosarcomas of the skull base. J Neurosurg 91: 432-439, 1999.

39. Chugh R, Tawbi H, Lucas DR, Biermann JS, Schuetze SM and Baker LH: Chordoma: the nonsarcoma primary bone tumor. Oncologist 12: 1344-1350, 2007. 